\title{
3x3 Optical Switch by Exploiting Vortex Beam Emitters based on Silicon Microrings with Superimposed Gratings
}

\author{
Mirco Scaffardi ${ }^{1, *}$ Muhammad N. Malik, ${ }^{1,2}$ Emma Lazzeri ${ }^{2}$, Charalambos \\ KLITIS $^{3}$, LAURA MeriggI ${ }^{3}$, Ning ZhANG ${ }^{3}$, MARC SOREL ${ }^{3}$, Antonella BogonI ${ }^{2}$ \\ ${ }^{1}$ CNIT, via G. Moruzzi 1, 56124, Pisa \\ ${ }^{2}$ Scuola Superiore Sant'Anna, Via Moruzzi 1, 56124 Pisa, Italy \\ ${ }^{3}$ University of Glasgow, Oakfield Avenue, Glasgow G12 8LT, UK \\ *Corresponding author: mirco.scaffardi@cnit.it
}

Received XX Month XXXX; revised XX Month, XXXX; accepted XX Month XXXX; posted XX Month XXXX (Doc. ID XXXXX); published XX Month XXXX

\begin{abstract}
A silicon-on-insulator microring with three superimposed gratings is proposed and characterized as a device enabling 3x3 optical switching based on orbital angular momentum and wavelength as switching domains. Measurements show penalties with respect to the back-toback of $<1 \mathrm{~dB}$ at a bit error rate of $10^{-9}$ for $00 \mathrm{~K}$ traffic up to 20Gbaud. Different switch configuration cases are implemented, with measured power penalty variations of less than $0.5 \mathrm{~dB}$ at bit error rates of $10^{-9}$. An analysis is also carried out to highlight the dependence of the number of switch ports on the design parameters of the multigrating microring. (C) 2017 Optical Society of America
\end{abstract}

OCIS codes: (130.3120) Integrated optics devices; (130.4815) Optical switching devices

http://dx.doi.org/10.1364/OL.99.099999

Optical switching can offer effective solutions to overcome the scalability issues of current electrical interconnection networks, which are limited by power consumption, power dissipation and footprint problems [1]. Specifically, interconnection networks scalability can be enhanced by operating over multiple switching domains such as space, wavelength, and time [2]. The orbital angular momentum (OAM) of light can represent a further domain to improve scalability and performance of the multilayer interconnection networks, since beams with different OAM orders are orthogonal [3]. Recent examples of this approach include a reconfigurable $2 \times 2$ OAM optical switch based on spatial light modulators (SLMs) [4] and 4x4 OAM switching with 2 SLMs [5]. However, SLM-based implementations are bulk and limited in terms of switching speed to the millisecond timescale.
Integrated devices such as microrings with super-imposed grating structures [6], circular grating couplers cascaded to star couplers [7] and hybrid 3D integrated circuits [8] have been demonstrated as compact alternatives to generating tunable and reconfigurable OAM beams. Such devices are attractive for the small form factor (i.e. tens of micrometers) and low-power consumption and fast tunability (i.e. tens of $\mathrm{mW}$ and $\mu$ s timescale with thermal tuning). In [9] we proposed a switching architecture exploiting OAM and wavelength as switching domains based on integrated OAM microring emitters.

Here we show that a Silicon-on-insulator microring with three superimposed gratings can be effectively employed as a device enabling $3 \times 3$ optical switching. The switch exploits simultaneously the OAM and wavelength as switching domains, allowing full reconfigurability without blocking states. We also carry out an analysis in order to clarify the dependence of the number of switch ports on two microring design parameters, i.e. the number of superimposed gratings and the number of elements of each grating.

The key element for the implementation of the switch is a microring resonator with $N_{g}=3$ superimposed gratings, each designed to emit a different OAM order. Specifically, the microring, with radius of $29 \mu \mathrm{m}$ and fabricated in standard Silicon-on-insulator (SOI) technology, has three overlapped gratings with different numbers of grating elements $q_{i}$. Each grating emits an OAM mode of order $l_{j}$ depending on the resonance peak at which the input wavelength is set. The gratings were designed so that the difference among the orders of the OAM modes emitted simultaneously by the three microrings is $\Delta q=q_{2}-q_{1}=q_{3}-q_{2}=3$, i.e. if $l_{j}$ is the order emitted by the first grating, $l_{j}+3$ and $l_{j}+6$ are the OAM orders emitted by the second and third grating, respectively. The motivation for this design choice will be motivated in the final discussion section. 
Table 1: Mapping of the OAM mode order $l$ emitted by the microring with three superimposed gratings $\left(N_{g}=3, \Delta q=3\right)$ vs. the wavelength $\lambda_{k}$. The labels $\mathrm{Si}$ indicates the sets of wavelengths available for each switch input port.

\begin{tabular}{|c|c|c|c|}
\hline & \multicolumn{3}{|c|}{ Order lof emitted OAM modes } \\
\hline Wavelength(nm) & Grating 1 & Grating 2 & Grating 3 \\
\hline$\lambda 1: 1536.91$ & 2 & 5 & $8(S 2)$ \\
\hline$\lambda 2: 1539.76$ & 3 & 6 & $9(S 3)$ \\
\hline$\lambda 3: 1542.58$ & 4 & 7 & $10(S 1)$ \\
\hline$\lambda 4: 1545.99$ & 5 & $8(S 3)$ & 11 \\
\hline$\lambda 5: 1548.65$ & 6 & $9(S 1)$ & 12 \\
\hline$\lambda 6: 1551.87$ & 7 & $10(S 2)$ & 13 \\
\hline$\lambda 7: 1555.04$ & $8(S 1)$ & 11 & 14 \\
\hline$\lambda 8: 1558.20$ & $9(S 2)$ & 12 & 15 \\
\hline$\lambda 9: 1561.28$ & $10(S 3)$ & 13 & 16 \\
\hline
\end{tabular}

The mapping between wavelengths and OAM orders shows that the set of OAM modes with $l=8,9,10$ is the most convenient as it can be generated by wavelengths in the $\mathrm{C}$ band for all three gratings (see Table 1).

The $3 \times 3$ OAM switch architecture is implemented by cascading the three-grating microring, which emits a set of three OAM modes depending on the wavelengths of the three input signals $S 1, S 2$, and $S 3$, with an OAM sorter as detailed in Fig. 1. The OAM modes emitted by the microring are multiplexed in a coaxial beam propagating along a direction orthogonal to the plane of the device. The passive OAM mode sorter allows the routing of the signal coming from each input $S i$ to a different spatial direction depending on the order of the incoming OAM mode, which therefore identifies the switch output port [9]. Since the triplet of wavelength associated to $S 1, S 2$ and $S 3$ is different, all the input signals can be directed to the same output port without interference and eventually be separated by optical wavelength filtering.

The $3 \times 3$ OAM switch is tested with the experimental setup shown in Fig. 2. The input signals are generated from three tunable lasers operating at different wavelengths and fed into a Mach-Zehnder amplitude modulator driven by an electrical PRBS 231-1. The signals are then decorrelated with single mode fibre spans (SMFi) and amplified to $25 \mathrm{dBm}$ at the input of the microring in order to compensate for the coupling losses. A SLM is used to detect a specific OAM mode and to convert it back to Gaussian for fibre coupling. The OAM mode conversion operation is performed independently of the signal wavelength. The signal is finally amplified, loaded with noise and filtered (BPF) before being received and monitored with an optical spectrum analyser (OSA). The total loss of the three-grating microring is about $16.8 \mathrm{~dB}(2 \mathrm{~dB}$ of insertion loss, $10 \mathrm{~dB}$ of emission efficiency and $4.8 \mathrm{~dB}$ of OAM mode splitting loss) and the bandwidth is about $0.2 \mathrm{~nm}$.

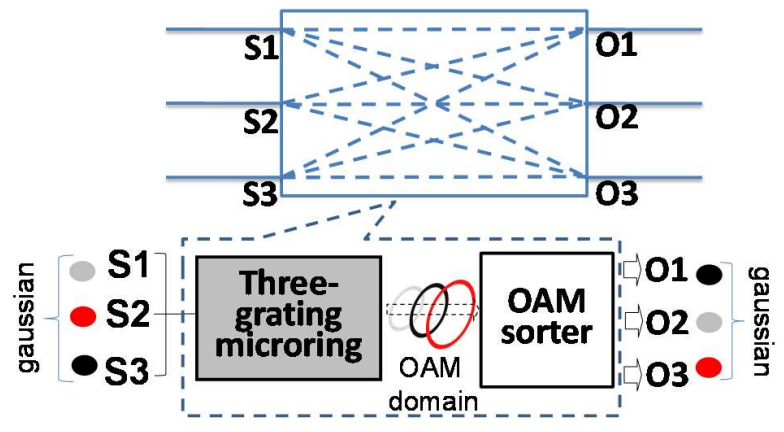

Fig. 1. Schematic of the $3 \times 3$ OAM-based switch exploiting a threegrating microring and an OAM mode sorter.

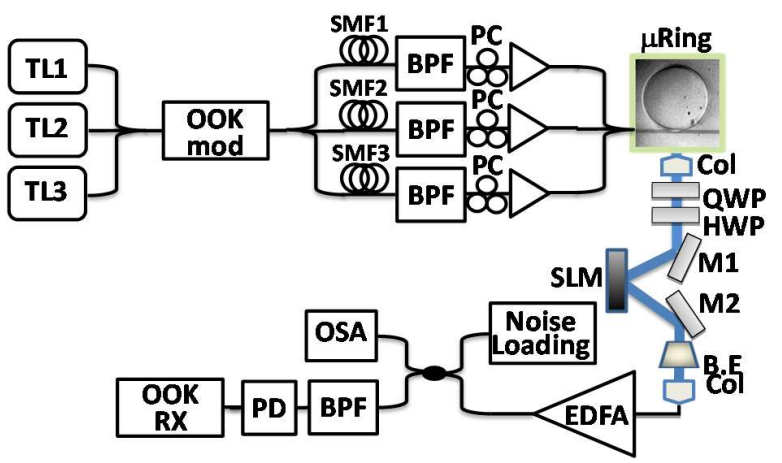

Fig. 2. Experimental setup for the OAM $3 \times 3$ switch characterization.

The input fiber-to-output fiber total loss is about $22.8 \mathrm{~dB}$. Further details on the multi-grating microrings and their exploitation can be found in [9]. It is worth noting that the OAM sorter can be implemented with different techniques, such as cascaded refractive elements [10], complex phase masks loaded on SLMs [11], and integrated demultiplexers [7-8]. In all these approaches the OAM beam is deflected and contemporary converted to Gaussian. OAM sorting without mode conversion to Gaussian is also possible by exploiting a cascade of Mach-Zhender interferometers based on Dove prisms [12]

The three-grating microring and a detail of the superimposed grating (taken at the scanning electron microscope) are shown in Fig. 3(a) and (b) respectively. The infrared image of the near field emitted by the microring is shown in Fig. 3 (c). The near field intensity presents minima corresponding to regions where the protrusion of the grating in the ring inner side wall is minimum. This is due to the superposition of three grating characterized by different period, which generates, as a linear combination of periodic functions, a periodic variation of the grating element protrusion along the circumference as shown in Fig. 3 (d) [6].

System performance is measured at 20 Gbaud in two different switching configurations, with the signals sent to a) different output ports and b) the same output port. In terms of modal crosstalk, the first configuration represents the less critical case with three wavelengths mapped onto three different OAM modes, while the second represents the most critical cases with three wavelengths mapped onto the same OAM mode. 

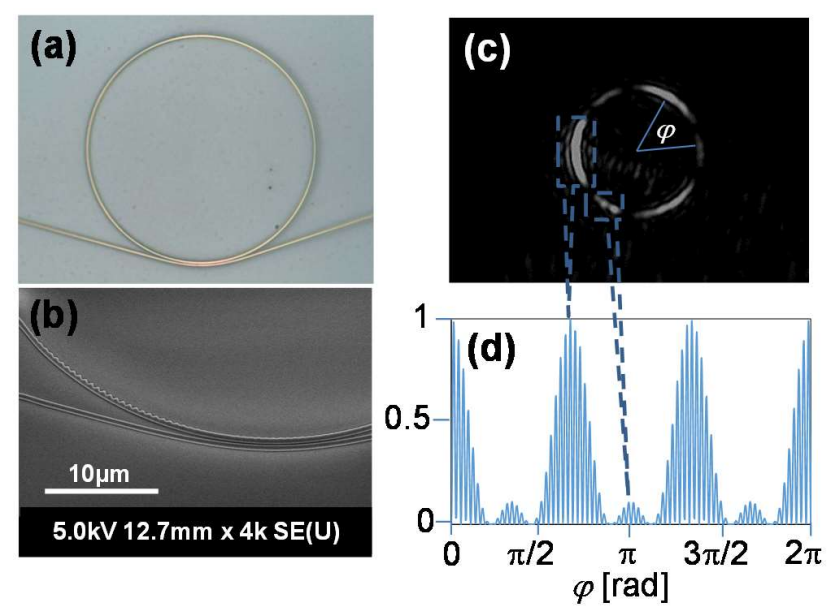

Fig. 3. (a) Image of the three-grating microring. (b) Detail of the superimposed gratings at the scanning electron microscope. (c) Infrared image of the near field emitted by the microring with three superimposed gratings. (d) Unfolded view of the squared grating perturbation function versus the azimuthal angle $(\varphi)$.The six intensity minima and the six intensity maxima present in (c) find correspondence with the six minima and maxima of the grating perturbation function (d).

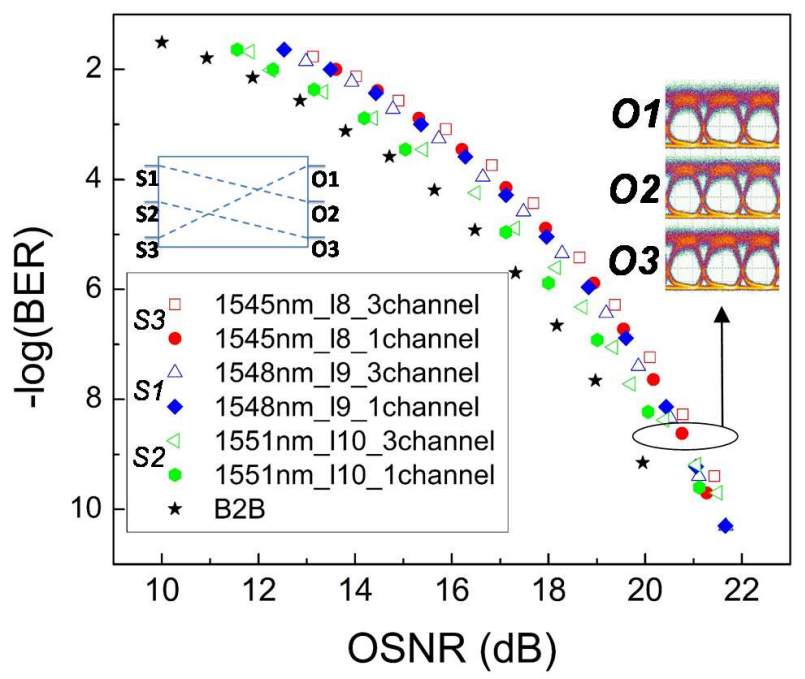

Fig. 4. BER vs. OSNR for the switch configured as follows: $S 1$ to $02, S 2$ to $03, S 3$ to 01 . The output eye diagrams at 20Gbaud are shown in the inset.

Fig. 4 shows the bit error rate (BER) vs. optical signal-tonoise ratio (OSNR) for configuration a), i.e. $S 1$ directed to $O 2$, $S 2$ to $03, S 3$ to 01 . At a $\mathrm{BER}=10^{-9}$ the OSNR sensitivity difference among the output signals is $<0.5 \mathrm{~dB}$ when all the three input channels are present. It is worth pointing out that there is negligible performance degradation with respect to the case where a single channel is present at the input, i.e. the microring performance does not depend on the number of input signals.

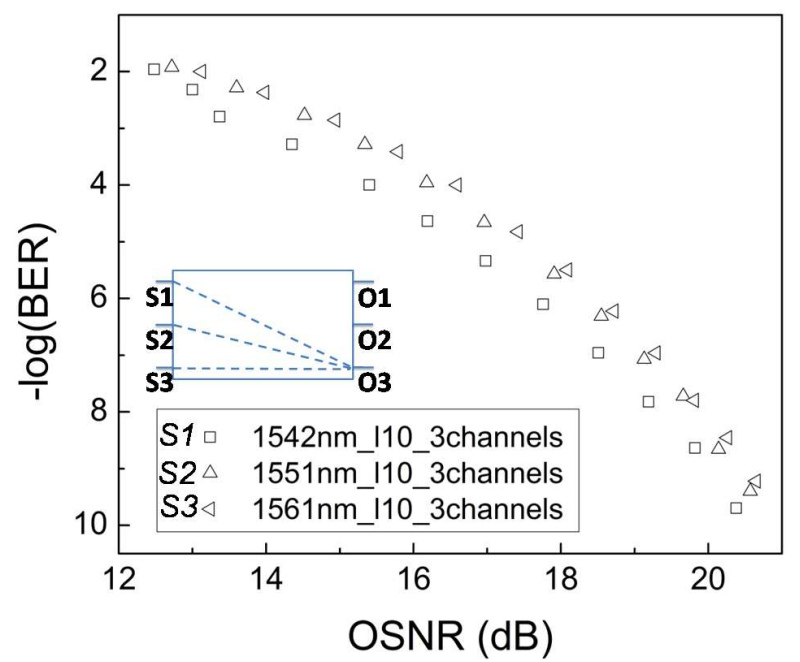

Fig. 5. BER vs. OSNR for the switch configured as follows: $S 1$ to $03, S 2$ to $03, S 3$ to 03 .

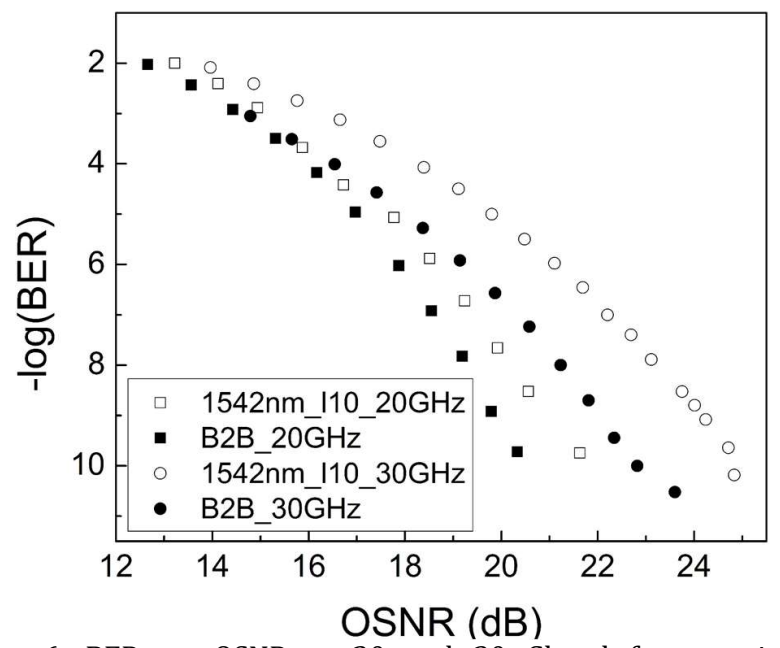

Fig. 6. BER vs. OSNR at 20 and 30 Gbaud for a switch configuration in which all three input signals are sent to the same output and the corresponding back-to-back curves.

The penalty with respect to the back-to-back, measured by replacing the microring with a filter of the same $3 \mathrm{~dB}$ bandwidth $(\sim 0.2 \mathrm{~nm})$, is $<1 \mathrm{~dB}$. The three insets show an open eye diagram for the three signals at the microring output.

Fig. 5 shows the BER vs. OSNR when all the input signals are sent to the same output port 03. An OSNR sensitivity difference $<0.5 \mathrm{~dB}$ is measured, i.e. there is no performance degradation with respect to the previous switch configuration. The good performance is favored by the wavelength diversity of the signals, which avoids interference. By increasing the baud rate up to 30 Gbaud, error-free performance can still be obtained, with an additional penalty of $3.5 \mathrm{~dB}$ (see Fig. 6). Since the multigrating microring is completely passive, the switching speed is limited by the wavelength tuning speed of the input signals. If the signals are generated with distributed feedback laser (DFB) or distributed Bragg reflector (DBR) laser, tuning speed in the microsecond range can be achieved $[13,14]$. 
Table 2: Mapping of the OAM mode order $l$ emitted by a device with $N g=3$ and $\Delta q=2$, and $N g=2$ and $\Delta q=4$ vs. the wavelength $\lambda_{k}$ of the resonant peaks.

\begin{tabular}{|c|c|c|c|c|c|}
\hline \multirow[b]{3}{*}{ Wav } & \multicolumn{5}{|c|}{$I$ (emitted OAM order) } \\
\hline & \multicolumn{3}{|c|}{$\Delta \mathrm{q}=2$} & \multicolumn{2}{|c|}{$\Delta q=4$} \\
\hline & G1 & $\mathrm{G} 2$ & G3 & G1 & G2 \\
\hline$\lambda 1$ & $l$ & $l+2$ & $\mathrm{l}+4$ & $l$ & $l+4$ \\
\hline$\lambda 2$ & $l+1$ & $l+3$ & $1+5$ & $l+1$ & $l+5$ \\
\hline$\lambda 3$ & $1+2$ & $l+4$ & $1+6$ & $l+2$ & $1+6$ \\
\hline$\lambda 4$ & $l+3$ & $l+5$ & $1+7$ & $l+3$ & $l+7$ \\
\hline$\lambda 5$ & $l+4$ & $l+6$ & $1+8$ & $l+4$ & $l+8$ \\
\hline$\lambda 6$ & $l+5$ & $l+7$ & $1+9$ & $l+5$ & $l+9$ \\
\hline$\lambda 7$ & $l+6$ & $l+8$ & $l+10$ & $l+6$ & $l+10$ \\
\hline$\lambda 8$ & $l+7$ & $l+9$ & $l+11$ & $1+7$ & $l+11$ \\
\hline$\lambda 9$ & $l+8$ & $l+10$ & $1+12$ & $l+8$ & $l+12$ \\
\hline
\end{tabular}

With particular laser structures as V-cavity lasers, tuning speed in the nanosecond range can be achieved [15].

The number of switch ports can be extended by increasing the number of gratings $N_{g}$ superimposed on the microring and the difference among the number of grating elements $\Delta q$. In the implemented $3 \times 3$ OAM switch we exploited a multigrating microring with $N_{g}=3$ and $\Delta q=3$. In Table 2 we show the mapping of the OAM mode order $l$ emitted by three- and two-grating microrings vs. the wavelength $\lambda_{k}$ of the resonant peaks for $\Delta q=2$ and 4 . We assume constant difference among the number of grating elements of the different superimposed gratings, i.e. $q_{1}-q_{2}=q_{2}-q_{3}=\Delta q$. Table 2 shows that when $N_{g}=3$ and $\Delta q=2$ two different OAM modes can be generated, each one on a set of three wavelengths, which allows to build a 3x2 OAM switch. When $N g=2$ and $\Delta q=4$ four OAM modes can be selected, each one on a set of two wavelengths, which enables the implementation of a $2 \times 4$ OAM switch.

The mapping reported in Table 2 confirms that the number $N$ of selectable OAM modes, i.e. the number of switch output ports, is $N=\Delta q$, while the number $M$ of selectable wavelengths for each mode, i.e. the number of input ports, is $M=N_{g}$. In order to build a switch with an equal number of input and output ports, the condition $\Delta q=N_{g}$ must be satisfied. The required tuning range for the wavelength of the input signals is a linear function of the microring free-spectral range, i.e. of the inverse of the microring radius. Wavelength division multiplexed (WDM) signals can also be managed in principle by the switch. If $K$ is the number of required WDM channels per input port, the number of allowed switch ports is $N_{g} / K$. Since wavelength tuning is required at the switch input to properly map the signals into the OAM mode to reach the destination port, wavelength conversion/tuning stages are also needed to change wavelength of the input WDM channels. In conclusion we proposed and characterized a silicon-oninsulator microring with three superimposed gratings as a device enabling $3 \times 3$ optical switching. The switch exploits orbital angular momentum and wavelength as switching domains. Bit error rate is measured for two different switch configurations, showing power penalty variation $<0.5 \mathrm{~dB}$ among the different cases and a penalty $<1 \mathrm{~dB}$ with respect to the back-to-back for 00K traffic up to 20Gbaud. We also carried out an analysis in order to highlight the dependence of the number of switch ports on the design parameters of the multigrating microring, i.e. the number of superimposed gratings and the number of grating elements.

Funding. This work has been funded by the Project ROAMRevolutionizing optical fiber transmission and networking using the orbital angular momentum of light (Call ID: H2020-ICT-2014-1; topic: ICT-06-2014; funding scheme: RIA; contract number: 645361) and by the project Cornerstone (EPSRC - EP/L021129/1). The authors acknowledge the support from the technical staff of the James Watt Nanofabrication Centre at Glasgow University.

\section{References}

1. H. Cho, P. Kapur, K.C. Saraswat, J. Light. Tech., 22, 2021 (2004).

2. I. Cerutti, N. Andriolli, P. G. Raponi, M. Scaffardi, O. Liboiron-Ladouceur, A. Bogoni, P. Castoldi, IET Optoelect., 6, 192 (2012)

3. . J. Wang, J-Y. Yang, I. M. Fazal, N. Ahmed, Y. Yan, H. Huang, Y. Ren, Y. Yue, S. Dolinar, M. Tur, A. E. Willner, Nat. Photonics, 6, 488,(2012).

4. N. Ahmed, H. Huang, Y. Ren, Y. Yan, G. Xie, M. Tur, A. E. Willner, Optics Express, 22,756 (2014)

5. J. Liu, J. Wang, Scientific Reports, srep37331, (2016).

6. Q. Xiao, C. Klitis, S. Li, Y. Chen, X. Cai, M. Sorel, S. Yu., Optics Express, 24, 3168 (2016).

7. N. K. Fontaine, C. R. Doerr, L. L. Buhl, OFC, OTu1I.2 (2012).

8. C. Quin, B. Guan, R. Scott, R. Proietti, N. K. Fontaine, T. Su, C. Ferrari, M. Cappuzzo, F. Klemens, B. Keller, M. Earnshaw, S. J. B. Yoo, OFC, Th4A (2014).

9. M. Scaffardi, M. N. Malik, E. Lazzeri, G. Meloni, F. Fresi, L. Poti, N. Andriolli, I. Cerutti, C. Klitis, L. Meriggi, N. Zhang, M. Sorel, A. Bogoni,, IEEE Journal of Light. Techn., 15, 3142 (2017).

10. H. Huang, G. Milione, M. P. J. Lavery, G. Xie, Y. Ren, Y. Cao, N. Ahmed, T. An Nguyen, D. A. Nolan, M.-J. Li, M. Tur, R. R. Alfano, A. E. Willner, , Scientific Reports, srep14931 (2015).

11. S. Li, J. Wang, Scientific Reports, srep15406 (2015).

12. M. P. J. Lavery, A. Dudley, A. Forbes, J. Courtial, M. J. Padgett, New Journal of Physics, 13, 093014 (2011)

13. V. Polo, P. Borotau, A. Lerin, J. Prat, ECOC, P.4.13 (2014).

14. K. Numata, J. R. Chen, S. T. Wu, Optics Express, 20, 14234, (2012).

15. X. Liao, J. Guo, J.-J. He, IEEE Photonics Journal, 4, 1503807 (2017). 